pigmentation and cuticular decorations in insects and vertebrates ${ }^{6-9}$. More frequently, morphological differences involve several major-effect genes as well as weaker modifiers ${ }^{8,10-12}$. The existence of major-effect genes makes the evolution of animal morphology much more tractable at the molecular level than it would be under a highly polygenic model.

\section{Hopeful monsters}

Lest we start believing in hopeful monsters ${ }^{13}$, we should keep in mind the distinction between major-effect genes and major-effect mutations. A single locus may exert a substantial effect on the phenotype in interspecific genetic crosses or in transgenic animals. But there is no reason to think that this difference appeared all at once as a single mutation event. Rather, a gradual accumulation of many mutations, each with only a slight effect on the phenotype, may eventually turn a locus into what we perceive, in retrospect, to be a major-effect gene. When Wang and

\author{
William E Evans \& R Kip Guy
}

Chamberlin compared the cis-regulatory sequences of lin- 48 between C. elegans and its relative species ${ }^{4}$, they found extensive differences, including multiple nucleotide substitutions and deletions that affect putative binding sites for Ces-2, an important upstream regulator of $l i n-48$ (ref. 5). We do not know which of these molecular changes were responsible for the new expression pattern acquired by lin- 48 in the C. elegans lineage, but we are probably looking at a cumulative effect of multiple mutations.

The existence of major-effect genes tells us more about development than about evolution. There may simply be a limited number of developmental mechanisms that an animal can use to achieve a given phenotype ${ }^{9,14}$. Only a close synthesis of developmental biology with evolutionary and population genetics can help us understand how evolution explores these limited possibilities. Groups of closely related species of worms and flies have become a fertile ground for this synthesis ${ }^{15}$ and will no doubt continue to produce exciting results.
1. Carroll, S.B., Grenier, J.K. \& Weatherbee, S.D. From DNA to Diversity: Molecular Genetics and the Evolution of Animal Design (Blackwell Science, Oxford, Malden, Massachusetts, 2001).

2. Wilkins, A.S. The Evolution of Developmental Pathways (Sinauer Associates, Sunderland, Massachusetts, 2002)

3. Davidson, E.H. Genomic Regulatory Systems: Development and Evolution (Academic, San Diego, 2001).

4. Wang, X. \& Chamberlin, H.M. Nat. Genet. 36, 231-232 (2004)

5. Wang, X. \& Chamberlin, H.M. Genes Dev. 16 2345-2349 (2002).

6. Nachman, M.W., Hoekstra, H.E. \& D'Agostino, S.L. Proc. Natl. Acad. Sci. USA 100, 5268-5273 (2003).

7. Theron, E., Hawkins, K., Bermingham, E., Ricklefs, R.E. \& Mundy, N.I. Curr. Biol. 11, 550-557 (2001).

8. Wittkopp, P.J., Carroll, S.B. \& Kopp, A. Trends Genet. 19, 495-504 (2003).

9. Sucena, E., Delon, I., Jones, I., Payre, F. \& Stern, D.L. Nature 424, 935-938 (2003)

10. Swalla, B.J., Just, M.A., Pederson, E.L. \& Jeffery, W.R. Development 126, 1643-1653 (1999).

11. Naisbit, R.E., Jiggins, C.D. \& Mallet, J. Evol. Dev. 5, 269-280 (2003).

12. Takano-Shimizu, T. Genetics 156, 269-282 (2000).

13. Goldschmidt, R. The Material Basis of Evolution (Yale University Press, New Haven, 1940).

14. Gompel, N. \& Carroll, S.B. Nature 424, 931-935 (2003).

15. Simpson, P. Nat. Rev. Genet. 3, 907-917 (2002).

\title{
Gene expression as a drug discovery tool
}

\begin{abstract}
After establishing a pattern of gene expression that identifies a desired drug effect in leukemia cells (e.g., differentiation), post-treatment gene expression can be used to screen candidate compounds for their ability to induce the target phenotype.
\end{abstract}

Most medications currently used to treat cancer are relatively nonspecific cytotoxic agents, exerting effects on both tumor and normal tissue. The treatment of childhood acute lymphoblastic leukemia, for which cure rates have increased from $<10 \%$ in the 1960 s to over $80 \%$ today $^{1}$, can be attributed to the use of cytotoxic chemotherapeutic agents with low specificity. Unfortunately, such success is not evident in many human cancers, including acute myeloid leukemia (AML), where $<50 \%$ of affected individuals are cured with cytotoxic chemotherapy alone. New selective agents are needed for essentially all human

William E. Evans is Scientific Director of St. Jude Children's Research Hospital, Memphis, Tennessee, USA, and R. Kip Guy is Director of the Bay Area Screening Center of the California Institute for Quantitative Biomedical Research at University of California San

Francisco, San Francisco, California, USA. e-mail: william.evans@stjude.org

orrguy@cgl.ucsf.edu malignancies, to further improve cure rates and reduce host toxicity.

Advances in our understanding of cancer biology, human genetics, disease pathogenesis and molecular pharmacology hold great promise for the discovery of new approaches to treating cancer ${ }^{2}$. Progress and expectations have been heightened by initiatives such as the human genome project and by advances in technology that permit genome-wide analysis of cancer cells and facilitate the synthesis and high-throughput screening of large libraries of candidate compounds against molecularly defined targets ${ }^{3}$. The predominant strategy for identifying new anticancer agents is to screen for compounds that interact with specific molecular targets, rather than screening for predefined post-treatment phenotypes. Mechanisms that induce such phenotypic differentiation are normally complex and almost always involve the combined interaction of multiple signaling pathways. Although there has been considerable growth in the use of methods with high information content, ${ }^{4}$ the ability to detect a particular phenotype during a primary screen has lagged behind the ability to measure the functions of single molecular targets.

\section{A new twist}

On page 257, Kimberly Stegmaier and colleagues report a new strategy for screening compounds that force myeloid differentiation of AML cells ${ }^{5}$. Their approach is based on the observation that all-trans retinoic acid produces clinical remissions by inducing differentiation of acute promyelocytic leukemias harboring a mutated retinoic acid receptor alpha ${ }^{6}$. They developed a surrogate marker approach that uses post-treatment gene expression signatures in AML cell lines as the read-out for screening the ability of candidate compounds to induce differentiation of AML cells (Fig. 1). Microarray-based approaches have been previously applied to determining the mechanism of action of small molecules $^{7-9}$ or their toxicology ${ }^{10}$ and to assessing hormonal effects ${ }^{11}$. But the logistical reality of 
attempting to run tens of thousands of microarray experiments has largely precluded their use as even a secondary screening technique in drug discovery. By comparing gene expression patterns in untreated primary AML cells and their differentiated myeloid counterparts from unaffected controls, the group identified the pattern of gene expression characteristic of the phenotypic transition. They then used statistical methods to identify a small subset of genes that were highly predictive of myeloid differentiation. The expression pattern of these five genes, as measured by mass spectrometry of the transcripts, was used to assess the ability of a library of $\sim 1,800$ candidate compounds to induce the differentiation expression signature in a human AML cell line. They identified 15 candidate compounds that induced the differentiation signature, 8 of which did so reproducibly. Two of the eight 'hits' have been previously shown, by more traditional methods, to induce myeloid differentiation. The hits were further classified by full geneexpression pattern analysis, observation of morphological changes and biochemical methods to show that these compounds induce myeloid differentiation in vitro. Of the eight hits, three scored well by all tests, indicating differentiation. The other five hits scored well on a subset of the phenotype tests, indicating that some compounds only partially induced the desired phenotype. In comparison to other screening methods, this is a good true-positive rate, and the utility of this method is confirmed by its ability to detect partial effects of some molecules.

\section{Eye on screening}

The reported method compares favorably with high-information content screening methods such as automated microscopy. Gene expression changes are measured directly as a read-out for signaling, whereas microscopy approaches typically rely on reporter assays or morphological changes that must be detected by automated image analysis. Thus, the new method should both provide the ability to detect subtle phenotypic changes that do not affect gross morphology and avoid artefactual signaling induced by reporters. The gene expression method will probably fall into the realm of secondary screening. But using mass spectroscopy as the detection method, thus avoiding gel electrophoresis or chromatography, potentially allows for scaling to the level of a primary screen with hundreds of thousands of compounds.

The mass spectrometry-based gene expression method is an extension of the use of gene

a

Definition of surrogate marker gene expression pattern

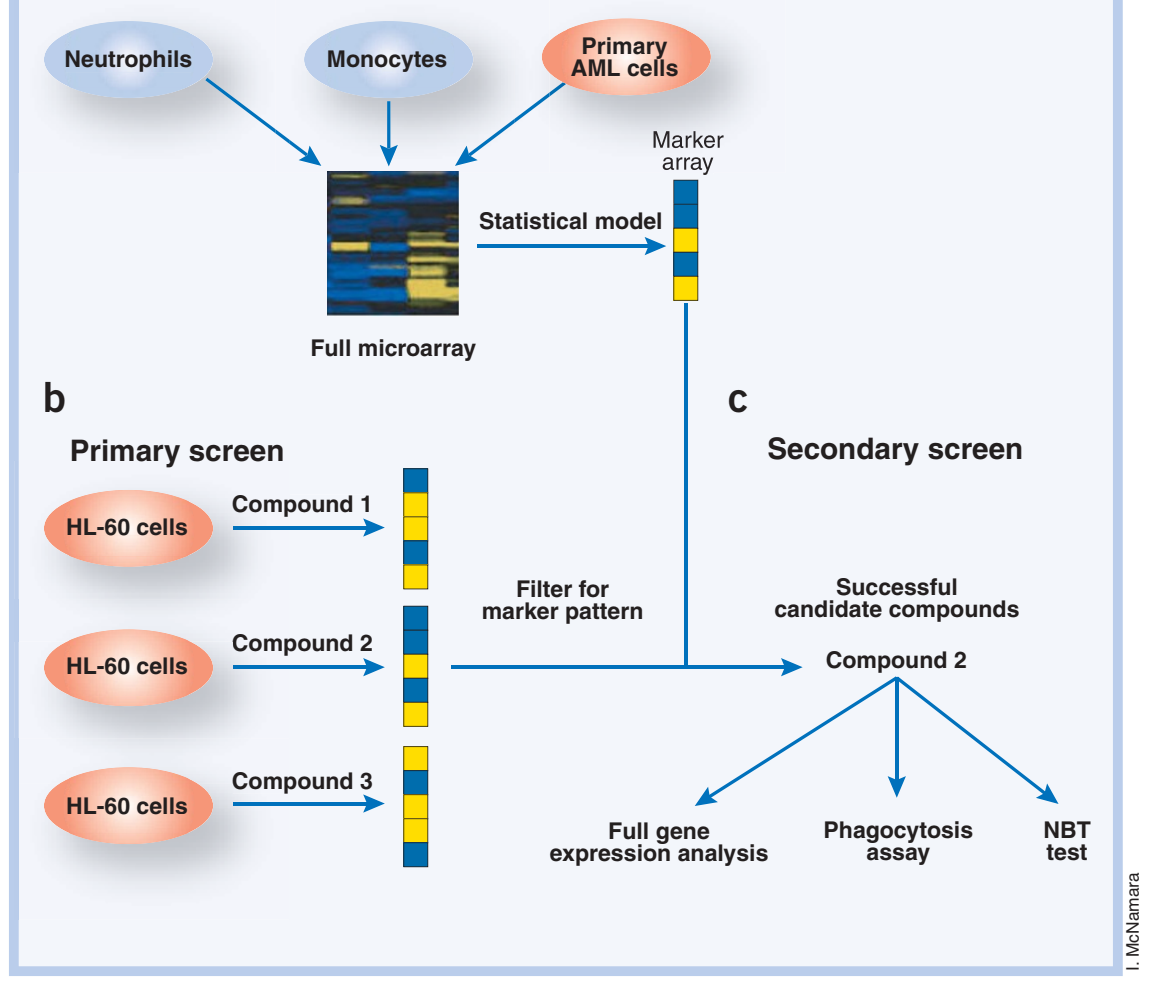

Figure 1 Gene expression as a read-out to identify candidate compounds that induce the desired cellular phenotype. (a) Establish a gene expression signature that identifies the desired phenotype in AML cells (e.g., differentiation). (b) Screen a library of compounds for induction of the target gene expression profile. (c) Validate successful compounds from the primary screen using additional assays.

expression as a tool for investigating the genomics of human cancer ${ }^{12,13}$, the effects of drug treatment ${ }^{8}$ and the basis of treatment failure $^{14}$. Chemotherapy has been shown to cause drug-specific changes in gene expression in acute lymphoblastic leukemia cells, discriminating the effects of single agents and drug combinations $^{13}$. The current work by Stegmaier and colleagues flips this model, by first defining the drug-induced pattern of gene expression that is the therapeutic goal and then screening compounds to find those that can induce this endpoint. In some ways, this can be viewed as a modern, mechanistically insightful, reimplementation of classical pharmacology, using a whole-cell response to drive drug discovery. The ultimate test, of course, will be in vivo data to illustrate that compounds identified by this approach will actually induce AML differentiation in preclinical models and ultimately make their way into clinical trials. The relatively low rate of emergence of such new compounds from conventional methods of drug discovery suggests that the identification of any bona fide inducers of AML differentiation by this strategy will represent a substantial advance in drug discovery for a disease that sorely needs more effective chemotherapy.

1. Pui, C.-H. \& Evans, W. N. Engl. J. Med. 339, 605-615 (1998).

2. Lindsay, M.A. Nat. Rev. Drug Discov. 2, 831-838 (2003).

3. Bleicher, K H., Bohm, H.J., Muller, K. \& Alanine, A.I. Nat. Rev. Drug Discov. 2, 369-378 (2003).

4. Yarrow, J., Feng, Y., Perlman, Z., Kirchhausen, T. \& Mitchison, T. Comb. Chem. High Throughput Screen. 6, 279-286 (2003).

5. Stegmaier, K. et al. Nat. Genet. 36, 257-263 (2004).

6. Huang, M. et al. Blood 72, 567-572 (1988).

7. Marton, M.J. et al. Nat. Med. 4, 1293-1301 (1998).

8. Cheok, M. et al. Nat. Genet. 34, 85-90 (2003).

9. Wilson, M. et al. Proc. Natl. Acad. Sci. USA 96, 12833-12838 (1999).

10. Waring, J.F., Ciurlionis, R., Jolly, R.A., Heindel, M. \& Ulrich, R.G. Toxicol. Lett. 120, 359-368 (2001).

11. Feng, X., Jiang, Y., Meltzer, P. \& Yen, P.M. Mol. Endocrinol. 14, 947-955 (2000).

12. Golub, T. et al. Science 286, 531-537 (1999).

13. Yeoh, E.-J. et al. Cancer Cell 1, 133-143 (2002).

14. Holleman, A. et al. Proc. Am. Assoc. Cancer Res. 44, 310 (2003). 